\title{
ERRATUM. I
}

To the article "Metaheuristic Downhill Simplex Method in Combinatorial Optimization" by L. F. Gulyanitskii and I. V. Sergienko, Vol. 43, No. 6, pp. 822-829, November-December, 2007.

The name of the first author was misspelled. It should read L. F. Hulianytskyi. 